\title{
Effectiveness of digital health using the transtheoretical model to prevent or delay type 2 diabetes in impaired glucose tolerance patients: protocol for a randomized control trial
}

Rasmieh Alzeidan ${ }^{1}$, Zeinab Shata ${ }^{2}$, Marwah Mazen Hassounah ${ }^{3}$, Leena Rashad Baghdadi ${ }^{4}{ }^{*}$, Ahmad Hersi ${ }^{1}$, Amel Fayed ${ }^{2,5}$, Tarek Kashour ${ }^{1}$ and Hala Elmorshedy ${ }^{2,5}$

\begin{abstract}
Background: There is high prevalence of prediabetes and type 2 diabetes mellitus (T2DM) in Saudi Arabia that is still increasing. Early diagnosis of prediabetes, and immediate, effective intervention is yet unestablished. Conventional health promotion approaches are used to educate prediabetic patients. Behavior modification is very effective in prediabetics to delay T2DM. Thus, the main objective of this study is to examine the effect of the new behavioral model, the Transtheoretical Model short messages (text 4 change) to modify lifestyle to prevent or delay the onset of T2DM, through promotion of a healthy diet and increased physical activity, in impaired glucose tolerance patients. Another objective is to estimate the impact of this model on markers of cardiovascular and metabolic risks as T2DM is one of the modifiable risk factors to prevent cardiovascular diseases.

Methods: This is a randomized controlled trial. One thousand and sixteen, eligible Saudi adults will be recruited from the Heart Health Promotion study (HHP), which was conducted at the King Saud University from July 2013 to April 2014. These adults were at a higher risk of developing T2DM within 2-3 years. The research team's database has a contact list and they will recruit individuals over $6-8$ weeks. All participants will be randomized at a 1:1 ratio into two groups, receive group education about lifestyle modifications and written information about diet and physical activity. Text 4 change SMS texts will be sent only to the intervention group. All participants will be assessed at baseline, 6, 12, 18, 24, 30, and 36 months for behavioral change using a World Health Organization (WHO) STEPS questionnaire and for glycated hemoglobin, biochemical and anthropometric measurements using standard methods.

Discussion: This new approach for promoting the importance of behavior modification in prediabetics is expected to delay and/or prevent the development of T2DM in Saudi Arabia, subsequently reducing the risk of cardiovascular morbidity and mortality too. Results from this study will promote an innovative and high-tech way to decrease the burden of cardiovascular diseases in Saudi Arabia.
\end{abstract}

Trial registration: International Standard Randomized Control Trial, registration number ISRCTN10857643. Registered 4 June, 2018.

Keywords: Transtheoretical model, Type 2 diabetes mellitus, Glycated hemoglobin, Cardiovascular diseases, Healthy lifestyle, Diet, Physical activity

\footnotetext{
* Correspondence: Ibaghdadi@ksu.edu.sa

${ }^{4}$ Department of Family and Community Medicine, King Saud University and

King Khalid University Hospital, Riyadh, Saudi Arabia

Full list of author information is available at the end of the article
}

(c) The Author(s). 2019 Open Access This article is distributed under the terms of the Creative Commons Attribution 4.0 International License (http://creativecommons.org/licenses/by/4.0/), which permits unrestricted use, distribution, and reproduction in any medium, provided you give appropriate credit to the original author(s) and the source, provide a link to the Creative Commons license, and indicate if changes were made. The Creative Commons Public Domain Dedication waiver (http://creativecommons.org/publicdomain/zero/1.0/) applies to the data made available in this article, unless otherwise stated. 


\section{Background}

Impaired glucose tolerance (IGT) and impaired fasting glucose (IFG) are intermediate conditions sometimes called prediabetes, where blood glucose levels are above normal but below diabetic thresholds [1]. Seventy percent of individuals with prediabetes eventually develop type 2 diabetes mellitus (T2DM) [2]. While people with IFG have a $20-30 \%$ chance of developing diabetes over the course of 5-10 years [3-5], this chance escalates to $90 \%$ if the individual is diagnosed with IGT [6]. In Saudi Arabia, the prevalence of both prediabetes and T2DM is high (18.3 and $11.9 \%$, respectively) and increases with age [7]. Even though T2DM is treatable, it is usually associated with other comorbidities such as hypertension, hypercholesterolemia and obesity. These conditions in patients with T2DM contribute to the development of cardiovascular diseases (CVD). Thus, T2DM is one of the modifiable risk factors that can decrease the risk of several chronic diseases such as CVD, and reduce its associated mortalities. A population-based study from England showed that prediabetes may convert back to normal glycemia [8]. Furthermore, landmark clinical trials have proven the effectiveness of lifestyle interventions towards a healthy diet and sufficient physical activity to reduce or delay the occurrence of T2DM by $50 \%$ in prediabetic individuals [9-11]. The Center For Disease Control and Prevention (CDC), and the American College of Sports Medicine have demonstrated that accumulation of 10,000 steps per day on most days of the week is an important component of improving glycemic control, insulin sensitivity, and/or cardiovascular (CV) risk in patients with T2DM $[1,12]$.

Targeting this at risk population of prediabetics and increasing their awareness about the importance of a healthy lifestyle will help in the long run, to significantly decrease the prevalence of T2DM in Saudi Arabia. While many educational programs have been used to promote health in the Saudi community, the use of a digital approach is still in its early stages [7]. Nowadays, the daily use of smartphones is vital due to their efficiency, connectivity and functionality. This makes the delivery of educational messages easier and more convenient.

Prediabetes complications have been associated with the long-term burden of CVD, which is a major cause of death among diabetic patients $[13,14]$. The risk of developing CVD increased 2-4 fold compared to age-sex matched patients without diabetes [15]. Worldwide, $43 \%$ of diabetes patients are more likely to die prematurely, and the rate of these deaths was higher in developing countries compared to developed countries [16]. Diabetes has a negative impact on the individuals' productivity and quality of life [17]. Diabetes also places a heavy economic burden on individuals, families, societies and countries. For example, globally, in 2017 diabetes consumed USD 727 billion from the health expenditure. In 2014, $14 \%$ of the total health expenditure of Saudi Arabia was utilized for diabetes management [18]. Recent studies showed that the prevalence of prediabetes among the general population in Saudi Arabia was $25.5 \%$ in 2014 [19], and $27 \%$ among university employees and their families in 2016 [20].

The 2018-2020 National Transformation Program delivery plan, one of the Kingdom's Vision 2030 programs, aims to improve health outcomes through e-Health. Over the last decade, the mobile phone short message service (SMS) has been increasingly used to deliver interventions designed to enhance healthy behavior [21]. SMS messages have the advantage of being inexpensive, convenient, reach the intended person instantly, and can be tailored to the individual [22]. In Saudi Arabia, the mobile phone market witnessed the fastest growth in the Middle East region with 23 million smartphones in use in 2017 [23].

Theory-led interventions base components of a health intervention on behavioral change, this theory has been constructed and called 'the Transtheoretical Model (TTM)' [24]. TTM has been used to improve adherence to medications in patients with CV risk factors $[25,26]$, and to prevent or delay the onset of T2DM in people with IGT [27, 28]. It has also been used effectively to predict or measure behavioral change in eating habits [29] and physical activity [30].

TTM posits that behavior change passes through five stages, which are precontemplation, contemplation, preparation, action, and maintenance (Table 1). These stages indicate the individuals' willingness to change their behavior ranging from long-term inactive (i.e. precontemplation stage) to long-term active (i.e. maintenance) behavior. Each stage has processes of change that are methods to move an individual from one stage of behavior to the other (Table 2) [24]. For example, raising consciousness (a process of change) is more effective with an individual in the precontemplation and contemplation stages rather than a person in the maintenance stage. Consequently, tailoring interventional messages based on the individual's stage of change would be more effective in progressing them to the next stage of change [32].

Therefore, this study aims to examine the effect of an interventional TTM based on short messages (text 4 change) of lifestyle modification to prevent or delay the onset of T2DM. The intervention will promote a healthy diet and increased physical activity among subjects with IGT. The design for this trial is parallel groups, the allocation ratio will be 1:1, and superiority framework.

\section{Primary objective}

To examine the effect of the TTM short messages (text 4 change) to modify lifestyle to prevent or delay the 
Table 1 Stages of change with brief descriptions

\begin{tabular}{ll}
\hline Stage of change & Description \\
\hline Precontemplation (PC) & $\begin{array}{l}\text { The individual is not willing to change in the foreseeable future (measured as the next } 6 \text { months). } \\
\text { Individuals in this stage are mostly uninformed or demoralized. } \\
\text { Contemplation (C) }\end{array}$ \\
$\begin{array}{l}\text { The individual is willing to change in the next } 6 \text { months. Individuals in this stage are aware of some } \\
\text { pros of behavior change but are still more inclined to value the cons. } \\
\text { The individual is willing to change in the foreseeable future (measured as the next month) and has } \\
\text { already taken some small steps towards change (in the past year). Individuals in this stage usually } \\
\text { have some plan on how to tackle this inactiveness. } \\
\text { The individual has changed, but not longer than } 6 \text { months. Individuals in this stage have 'changed' } \\
\text { but have not reached the duration which exemplifies real behavior change. }\end{array}$ \\
$\begin{array}{l}\text { Maintenance (M) } \\
\text { The individual has changed, longer than } 6 \text { months. Individuals in this stage have changed and are } \\
\text { working not to relapse. }\end{array}$ \\
\hline
\end{tabular}

onset of T2DM, through promoting a healthy diet and increased physical activity among subjects with IGT.

\section{Secondary objectives}

To estimate the impact of the TTM-based SMS messages (text 4 change) on regulating the level of biomarkers for cardiovascular and metabolic risks, lowering the indices of general and central obesity, and maintaining body measurements among nonobese individuals. To estimate the impact of the intervention program on the incidence of myocardial infarction, stroke, death and hospitalization. To examine the acceptability of the text
4 change motivational SMS messages among the intervention group.

\section{Methods/design}

Study design (setting, eligibility and recruitment)

This is a three-year randomized controlled trial that includes Saudis $\geq 18$ years of age. Participants for the present study will be recruited from the Heart Health Promotion study (HHP), which was conducted at King Saud University from 8 July, 2013 to 30 April, 2014 [20]. In the HHP study, participants were recruited from the three primary health care clinics serving the employees and their families at King Khalid University

Table 2 Processes of change in terms of experimental and behavioral processes (a brief description)

\begin{tabular}{|c|c|c|}
\hline Process & Description & \\
\hline \multicolumn{3}{|l|}{ Experiential processes } \\
\hline Consciousness raising (CR) & $\begin{array}{l}\text { The individual seeks increased knowledge about the causes, } \\
\text { consequences and cures for their problem behavior. }\end{array}$ & $\begin{array}{l}\text { From precontemplation } \\
\text { to contemplation }\end{array}$ \\
\hline Dramatic relief (DR) & $\begin{array}{l}\text { The individual's emotions about the problem behavior and } \\
\text { possible solutions are evoked. }\end{array}$ & \\
\hline $\begin{array}{l}\text { Environmental reevaluation } \\
\text { (ER) }\end{array}$ & $\begin{array}{l}\text { The impact that the individual's problem behavior has on } \\
\text { their environment is reevaluated. }\end{array}$ & \\
\hline Social liberation (SOL) & $\begin{array}{l}\text { Attempts are made to increase alternatives for the individual's } \\
\text { former problem behavior. }\end{array}$ & \\
\hline Self-reevaluation (SR) & $\begin{array}{l}\text { Cognitions and emotions regarding the individual with respect } \\
\text { to their problem behavior are reevaluated. }\end{array}$ & $\begin{array}{l}\text { From contemplation to } \\
\text { preparation and action }\end{array}$ \\
\hline \multicolumn{3}{|l|}{ Behavioral processes } \\
\hline Self-liberation (SEL) & $\begin{array}{l}\text { The individual has the belief that he can change and commits } \\
\text { to it by choosing a course of action. }\end{array}$ & \\
\hline Helping relationships (HR) & $\begin{array}{l}\text { The individual seeks trust and open discussion about the problem } \\
\text { behavior as well as support for the healthy behavior change. }\end{array}$ & Action to maintenance \\
\hline Counter-conditioning (CC) & $\begin{array}{l}\text { The individual substitutes positive behaviors for the individual's } \\
\text { problem behavior. }\end{array}$ & \\
\hline $\begin{array}{l}\text { Reinforcement management } \\
\text { (RM) }\end{array}$ & $\begin{array}{l}\text { Steps or changes made by the individual are rewarded when in a } \\
\text { positive direction or punished when in a negative direction. }\end{array}$ & \\
\hline Stimulus control (SC) & $\begin{array}{l}\text { Stimuli that may cue a lapse back to the problem behavior are } \\
\text { avoided and prompts for healthier alternatives are inserted. }\end{array}$ & \\
\hline Adapted from reference [31] & & \\
\hline
\end{tabular}


Hospital [20]. The HHP study was conducted on a large group of Saudi adults, who were at a higher risk of developing T2DM within 2-3 years. Recent data record updates of the original study on May 2017, revealed that out of the total sample (1234 prediabetic subjects) enrolled in the HHP study, 218 individuals had developed overt diabetes during the last 4 years (May 2013-May 2017). Therefore, 1016 are eligible for the present study.

\section{Inclusion criteria}

The inclusion criteria for participants is Saudi adults aged $\geq 18$ years, literate, prediabetic, with no contraindication of physical activity, have a smartphone with easy access to the internet and are willing and able to provide written informed consent to participate in the study.

\section{Exclusion criteria}

The exclusion criteria for participants is pregnancy, individuals who do physical exercise $>50 \mathrm{~min} /$ week, follow a diet regimen, have a history of myocardial infarction, have impaired renal function, and are diabetic.

\section{Recruitment}

Recruitment will take place over a 2-month period in order to ensure that all eligible subjects (from the HHP study) who fulfil the inclusion criteria are invited, give consent, and are enrolled following the steps of the baseline visits as shown in the flowchart (Fig. 1). The research team's database has a contact list and they will recruit individuals over 6-8 weeks. Subsequently, they will be given a 6-month follow-up visit appointment.

\section{Randomization and blinding}

Recruited, eligible participants will be randomized into two groups (1:1 ratio), using random numbers table generated in the Statistical Package for Social Studies (SPSS v. 21) (SPSS v. 21, IBM Corp., New York, NY, USA) by an independent statistician. The laboratory staff, biostatistician, health educator, and principal investigator will be blinded to the participant's group allocation until the end of the study. The participants will not be blinded to the intervention due to its nature, as well as the research assistant/project manager who is responsible for disseminating the text message intervention.

\section{Baseline visit}

\section{Lifestyle education}

Eligible prediabetic subjects from the previous HHP study [20] will be invited to the HHP office. If they give consent for this trial, then participants will be assigned to either the intervention or the control group by the computer-generated list. At this baseline visit, all participants will receive group education and motivation about lifestyle modifications along with written information about diet and physical activity recommendations offered by a diabetic educator and a physician. This will advise participants on balancing food intake and physical activity to achieve and maintain appropriate body weight. All participants will take Metformin $500 \mathrm{mg}$ twice daily. In addition, only participants in the intervention group will receive SMS messages at frequent intervals. These messages will be about a healthy lifestyle, the benefits of physical activity and a healthy diet. Neither group will receive any additional lifestyle information or advice by personal contact after the baseline visit, except in response to specific queries from the participants.

\section{Physical measurements}

At the baseline visit, all recruited participants' weight, height, waist circumference, and blood pressure from the left arm will be measured in a sitting position, and the mean of two readings will be considered. Blood tests for HbA1c and lipid profiles will be performed by qualified laboratory personnel. Glycated hemoglobin (HbA1c) and fasting lipid profiles will be measured for all participants at baseline and at every 6-month follow-up visit.

\section{Assessment of interventions}

All participants will complete the WHO STEPS instrument version 3.1 at the baseline visit and during each follow-up visit. This will assess behavioral risk factors including healthy diet, physical activity and the participants' behavioral change(s) stage based on the TTM at the baseline visit and follow-up visits for progression in the stages of change.

Data about adherence to physical activity and healthy dietary intake recommendations will be recorded at every 6-monthly follow-up visit. Adherence will be self-reported every 6 months and scored as poor, moderate or good. Physical activity and dietary intake will be categorized as adherent or nonadherent for statistical analysis.

At every visit after the baseline visit, we will assess the mobile messages in the intervention group through a specially designed questionnaire. It will assess the user acceptability and satisfaction of the SMS messages program using a scoring system of $0-10$. It will assess message content, frequency, clarity, effect on personal life ("degree of disturbance"), and personal views of the impact of these messages on lifestyle. The list of questions also includes the preferred time to receive messages and invites suggestions for improvement. A maximum total score of 10 denotes that the text messages were highly acceptable and satisfactory, while the lowest score of 
Flowchart of the work plan and collected data

\begin{tabular}{|c|c|c|}
\hline & Intervention group & Control group \\
\hline 1. & Consented subject & 1. Consented subject \\
\hline 2. & $\begin{array}{l}\text { Subjects assigned to the intervention group as per the } \\
\text { randomization list }\end{array}$ & $\begin{array}{l}\text { 2. Subjects assigned to the control group as per the } \\
\text { randomization list }\end{array}$ \\
\hline 3. & $\begin{array}{l}\text { Receive group education on standard lifestyle } \\
\text { recommendations }\end{array}$ & $\begin{array}{l}\text { 3. Receive group education on standard lifestyle } \\
\text { recommendations }\end{array}$ \\
\hline 4. & Start Metformin $500 \mathrm{mg}$ twice daily & 4. Start Metformin $500 \mathrm{mg}$ twice daily \\
\hline 5. & Fill WHO STEPS questionnaire & 5. Fill WHO STEPS questionnaire \\
\hline 6. & $\begin{array}{l}\text { Classify participants according to the TTM stage from } \\
\text { a question added to the questionnaire }\end{array}$ & $\begin{array}{l}\text { 6. Classify participants according to the TTM stage } \\
\text { from a question added to the questionnaire }\end{array}$ \\
\hline 7. & $\begin{array}{l}\text { Measuring weight, height, waist circumference and } \\
\text { blood pressure by research team }\end{array}$ & $\begin{array}{l}\text { 7. Measuring weight, height, waist circumference and } \\
\text { blood pressure by research team }\end{array}$ \\
\hline 8. & Blood tests for HBA1c and lipid profile & 8. Blood tests for HBA1c and lipid profile \\
\hline 9. & $\begin{array}{l}\text { Provide the research team with their steps count at the } \\
\text { first week of the } 6 \text {-month visits }\end{array}$ & $\begin{array}{l}\text { 9. Provide the research team with their steps count at } \\
\text { the first week of the } 6 \text {-month visits }\end{array}$ \\
\hline & Complete the messages evaluation form & 10. Complete the messages evaluation form \\
\hline
\end{tabular}

$1^{\text {st }}$ 6-month follow-up visit

1. Complete the WHO STEPS questionnaire including question on TTM stage of change

2. Measuring weight, height, waist circumference and blood pressure by the research team

3. Blood tests for HBA1c and lipid profile

$$
\begin{aligned}
& \text { 1. Complete the WHO STEPS questionnaire } \\
& \text { including question on TTM stage of change } \\
& \text { 2. Measuring weight, height, waist } \\
& \text { circumference and blood pressure by the } \\
& \text { research team } \\
& \text { 3. Blood tests for HBA1c and lipid profile }
\end{aligned}
$$

$2^{\text {nd }} \mathbf{6}$-month follow-up visit

Same steps as first 6-month visit

$3^{\text {rd }}$ 6-month follow-up visit

Same steps as first 6-month visit

$4^{\text {th }}$ 6-month visit follow-up visit

Same steps as first 6-month visit

$5^{\text {th }}$ 6-month visit follow-up visit

Same steps as first 6-month visit

$6^{\text {th }}$ Last 6-month visit

Same steps as first 6-month visit

Fig. 1 Flowchart for the work plan and data collection

zero denotes that the text messages were the least acceptable and unsatisfactory.

\section{Six-monthly follow-up visits}

Subjects will be given appointments for follow-up visits at the baseline visit, $6,12,18,24$ and 30 months, a total of six visits plus the baseline visit. Therefore, all subjects will repeat steps $1-3$ of the first 6-month follow-up visit, at each of these visits as shown in the flowchart (Fig. 1).

\section{Definitions and measurements} Definitions of cardiometabolic risk factors

Prediabetes [Impaired glucose tolerance (IGT) and impaired fasting glucose (IFG) [33, 34]] Subjects will be defined as prediabetic, if the level of glycated hemoglobin (HbA1c) is $5.7-6.4 \%$ as per WHO and American Diabetes Association (ADA) criteria [35], or the subject was previously diagnosed as prediabetic. 


\section{Diabetes mellitus}

Diabetes mellitus will be defined as per WHO and ADA criteria of $\mathrm{HbA} 1 \mathrm{c}$ level $\geq 6.5 \%$ or the subject being previously diagnosed as diabetic and using anti-diabetes medication [35].

\section{Dyslipidemia}

Subjects will be categorized as having any sort of dyslipidemia according to the $\mathrm{WHO}$ and the Third Adult Treatment Panel (ATP-III) of the National Cholesterol Education Program (NCEP). Dyslipidemia includes raised levels of total cholesterol, and/or low-density lipoprotein cholesterol (LDL-C), and/or triglycerides and low levels of high-density lipoprotein cholesterol (HDLC), or if the subject reported using medications to lower blood lipid levels [36, 37].

\section{Metabolic syndrome (MetS)}

Participants will be identified as having MetS using the NCEP-ATPIII criteria, if the subject has at least three out of five factors including abdominal obesity, dyslipidemia (raised triglycerides and reduced HDL-C), raised fasting plasma glucose and hypertension [38].

\section{Physical activity}

Participants will be identified as physically active if they achieve $150 \mathrm{~min}$ per week (at least 5 days per week) of moderate activity such as brisk walking or 1 hour of vigorous activity [39]. Furthermore, in the current study, we will measure their achieved steps per week, considered in the 1st week of each 6-month follow-up visit using a pedometer through health applications in the smartphones to count steps.

\section{Measurements of physical activity adherence}

Physical activity adherence was classified into three. Poor: Less than $150 \mathrm{~min} /$ week of moderate activity or 1 hour of vigorous activity (non-adherent). Fair: About 150-250 min/week of moderate activity or $1 \mathrm{~h}, 130 \mathrm{~min}$ of vigorous activity (adherent).

Good: More than $250 \mathrm{~min} /$ week of moderate activity, or if work involved, vigorous work (adherent).

\section{Healthy eating}

Healthy eating such as following the food pyramid and encouraging participants to consume the recommended portions of fruit and vegetables and avoiding unhealthy dietary habits, according to the WHO recommendations for a healthy diet [40]. This includes avoiding fast food, fatty food, sugar-sweetened beverages and refined carbohydrates, decreasing salt intake $(<5 \mathrm{~g} /$ day equivalent to sodium intake of $<2 \mathrm{~g}$ per day), encouraging more fiberrich food intake such as whole grains and legumes, and avoiding late night snacks.

\section{Measurements of dietary adherence}

Dietary adherence is divided into three categories: Poor: Not following the advice for more than five servings/ day/week (non-adherent). Fair: Occasional deviation from following advice for 2-4 servings/day/week (adherent). Good: Strictly following diet advice for more than 5 servings/day/week (adherent).

\section{Healthy weight and anthropometric measurements}

Participants will be classified according to WHO cutoff values for body mass index (BMI). Normal weight, overweight, obese and morbidly obese categories have a BMI of $18.5-24.9 \mathrm{~kg} / \mathrm{m}^{2}, 25-29.9 \mathrm{~kg} / \mathrm{m}^{2}, 30-34.9 \mathrm{~kg} / \mathrm{m}^{2}$ and $\geq$ $35 \mathrm{~kg} / \mathrm{m}^{2}$, respectively [41].

\section{Waist circumference}

The waist circumference criteria used for diagnosis of abdominal obesity in the Arab and Middle Eastern population is $\geq 88 \mathrm{~cm}$ and $\geq 102 \mathrm{~cm}$ for women and men, respectively $[37,42]$.

\section{Behavioral change}

As per the TTM [24], participants will be classified into five stages of changes (precontemplation (PC), contemplation (C), preparation, action, and maintenance) (Table 1) at the baseline visit and then every month during the first 6-month follow-up visit, through the WhatsApp application in the participants' smartphones. This will test whether the sent messages were appropriate for the stage of behavioral change. This evaluation will be updated based on self-reported questions, which will be given to the participants separately and sent back to the research team through the WhatsApp application.

\section{Intervention}

Interventions for this study will be on two levels. The standard health care, which is offered by primary health care services at King Saud University, Riyadh, Saudi Arabia and the text 4 change SMS messages.

\section{Message development}

The content of the messages is derived from the WHO resources for a healthy diet, [31] physical activity and diabetes, Saudi National Diabetes Prevention and Control Program educational material [43], studies with similar interventions, online social listening, and clinical and personal experiences of Saudi culture [44]. The messages will be in formal Arabic with the infusion of some phrases and words from the common Saudi dialect. Messages are written in the second person, using you, your, and yours. Each stage of change of the TTM has a designated number of messages to promote a healthy diet and physical activity. The messages' content was created according to the specific processes of change for 
each TTM stage. A total of 371 Arabic messages will be created by a health communication specialist and the project manager, who is well-acquainted with the target audience from a previous study. The specialist is wellacquainted with the Arabic speaking target population's local accent, sentiments and culture. The messages' content is based on international and national credible providers of health information and will be jointly prepared with a team of certified health educators. The messages will undergo cycles of feedback by a public health mental health specialist, a language reviser, and non-study participants from the target audience before the actual launch.

In the early stages of change (PC and $\mathrm{C}$ stages) that mainly require raising awareness, and contain nearly all essential messages needed to take the decision of change, every two messages are followed by a reminder, conveying the information mentioned in the previous messages every week (three for physical activity and three for a healthy diet). Reminders may take the form of text messages or multimedia messages (e.g. videos and infographics). For the following stages of change, where action is expected in the very near future or happening, the three weekly messages (for a healthy diet and physical activity) focus mainly on helping participants make a smooth transition through the process of change by providing a variety of messages conveying different alternatives of the same idea to achieve the goal of the stage.

The number of messages to cover 6 months are distributed as:

\section{Healthy diet messages}

Healthy diet messages were 189 in total, distributed per stage as in precontemplation, contemplation, preparation, action and maintenance, 55, 48, 14, 60 and 12, respectively (Table 3 ).

\section{Physical activity messages}

For physical activity, a total of $\mathbf{1 9 3}$ messages were created to cover the stages of change, namely precontemplation, contemplation, preparation, action, and maintenance, 56, 51, 14, 60 and 12, respectively (Table 3 ). The content covers the different processes of change (such as consciousness raising, helping relationships etc.) as suitable for each stage of change.

\section{Length and delivery method of the messages}

The SMS phone messaging will be done through the King Khalid University Hospital commercial delivery manager website (Easy SMS manager) and sent to participants three times per week during working hours. Messages will most probably be sent in the morning between 10 and $11 \mathrm{am}$. On each assigned day, they will receive two messages (one for physical activity and the other for diet) according to their stage of change. The easy SMS manager allows a message length of 70 Arabic language characters. If this limit is exceeded, it will be counted as two messages instead of one, thereby increasing the cost. The system uses a standardized communication protocol assuring the delivery of text messages to the participants; however, receiving text messages from the participants or exchanging messages is not permitted (i.e. it is a one-way system) To ensure that messages were delivered, they will also be sent to one of the authors' smartphones. At every visit, each participant will be asked about the number of messages received to ensure that all messages have been delivered and seen.

\section{Time of messaging}

The messages will be sent to the intervention group only in the first 6 months from the baseline. The following time points measure the intervention's long-term effect on biological markers, and the stages of change for healthy eating and physical activity.

\section{Measuring the TTM stage of change, the effect of intervention in the long term}

The TTM stage of change for physical activity and a healthy diet is determined at the baseline using a question adjunct to the WHO STEPS questionnaire. At the baseline visit and every month, the adjunct questions will be delivered to the participants' smartphone through the WhatsApp application. This will detect progress in the stage of change and help us move on to the next set of messages accordingly. The WHO STEPS questionnaire will be

Table 3 Number of messages according to the stage of change

\begin{tabular}{|c|c|c|c|c|c|c|}
\hline \multicolumn{7}{|c|}{ Healthy diet messages according to the stage of change } \\
\hline${ }^{\text {a Stages of change as per }{ }^{\mathrm{b}} \mathrm{TTM}}$ & Pre-contemplation & Contemplation & Preparation & Action & Maintenance & $\overline{\text { Total }}$ \\
\hline Number of messages & 55 & 48 & 14 & 60 & 12 & 189 \\
\hline \multicolumn{7}{|l|}{$\begin{array}{l}\text { Physical activity messages according } \\
\text { to the stage of change }\end{array}$} \\
\hline Number of messages & 56 & 51 & 14 & 60 & 12 & 193 \\
\hline Total & 111 & 99 & 28 & 120 & 24 & 382 \\
\hline
\end{tabular}

${ }^{\mathrm{a}}$ For each stage, three messages about diet and three messages about physical activity will be sent every week

${ }^{\mathrm{b}} \mathrm{TTM}$, Transtheoretical model of change 
administered at every 6-month follow-up visit to measure the interventions' long-term effects on biological markers and changes towards a healthy diet and physical activity.

\section{Acceptance of the text 4 change SMS}

At the first 6-month follow-up visit, we will assess the interventional messages using a specially designed questionnaire (adjunct questions). It will assess the user's acceptance and satisfaction of the SMS program, using a scoring system from 0 to 10 . It will assess the messages' content, frequency, clarity and personal view of the impact of messages on the participants' lifestyle. The list of questions also includes the preferred time to receive messages, and invites suggestions for improvement. A total score of 10 denotes that the text messages were highly accepted and satisfactory, and zero denotes that text messages were the least accepted and satisfactory.

\section{Pilot study}

Prior to commencing the actual study, we will recruit 30 non-study participants for a pilot study to be conducted in 1 month. We will assess the questionnaire for face validity, feasibility, and an estimate of the time taken to administer the questionnaire. Participants will be asked for verbal feedback while completing the survey. The SMS messages will be sent, based on the stage of change for each participant to review (from 408 messages) and give feedback on the ease of language and content. We will keep a feedback log to track all needed changes for the questionnaire and text messages. In this one-month pilot study, we will test the electronic messaging system, the feasibility of remote follow-up, and the documentation and data management strategy. Data collected in this pilot study will be analyzed and published.

\section{Study outcomes}

By the end of the trial, we expect the following outcomes:

\section{Primary outcome}

Adoption of healthy lifestyle behaviors including healthy eating and physical activity practices complying with WHO recommendations. Incidence of T2DM, based on a change in $\mathrm{HbA} 1 \mathrm{c}$ with an expected reduction of $1-2 \%$ at the end of the trial.

\section{Secondary outcomes at 6-month follow-up visits}

Change in the TTM towards stages of action and maintenance, decreased levels of LDL-C to $<130 \mathrm{mg} /$ $\mathrm{dl}$ or $\leq 5.1 \mathrm{mmol} / \mathrm{l}$, change in the systolicldiastolic blood pressure to normal blood pressure $<120 / 80$ $\mathrm{mmHg}$, respectively, decrease in body weight by $5-$ $10 \%$ per year, improvement in the physical activity level to the recommended $150 \mathrm{~min}$ per week and improvement in the daily consumption of portions of fruit and vegetables to 5-7 servings/day.

\section{Data analysis plan}

Data will be analyzed using the Statistical Package for Social Studies (SPSS 22; IBM Corp., New York, NY, USA). Continuous variables will be expressed as mean \pm standard deviation or median and interquartile ranges according to the normality assessment. Categorical variables will be expressed as frequencies and percentages.

The chi-square statistical test will be applied for categorical variables to assess the association between them. The t-test or Mann-Whitney test will be used to compare numeric variables. Repeated measures analysis of variances or Friedman's test will be used to evaluate repeated measures over the follow-up period.

The incidence rate and cumulative incidence rate will be computed for incidence of T2DM among the intervention and control groups. Additionally, comparison between the incidence of T2DM among different age groups and gender will be evaluated. Kaplan-Meier survival curves and log-rank tests will be used to compare the intervention and control groups as well as the different risk groups for progression to T2DM, and transition and/or relapses from different stages of TTM.

Hazards ratios with $95 \%$ confidence intervals will be calculated and the adjustment of known confounders will be adopted in the Cox regression model. In all the analyses, $P$-values $<0.05$ will be considered statistically significant.

The principal analyses of primary and secondary outcomes will use the intent-to-treat approach. The intentto-treat analyses will include all participants in their randomly assigned treatment groups regardless of a participant's adherence to the assigned treatment regimen.

All data entry will be anonymous and identification numbers will be saved in a password-protected log file with the primary investigator. Backup of entered data will be created monthly to avoid any loss of collected data. An interim analysis of data will be conducted 12 months after recruitment of the last participants. Interim analysis aims to evaluate the completeness and accuracy of the collected data and early detection of any difficulties.

\section{Sample size calculation}

A sample size of 300 is required so that there are 150 participants each in the intervention and control groups. The sample size was calculated by using Stata ${ }^{\circ}$ software version 15 (StataCorp, College Station, Texas, USA) with the assumptions that $\alpha=$ level of significance (0.05), $\beta=$ Type II error (power of the study $=1-\beta=80 \%$ ). The hazard rate of diabetes development among the control group is $10 \%$ over the 2 years, the hazard rate of diabetes development 
among the intervention group is $7 \%$ over the 2 years (reduction of about $30 \%$ ) and the minimum sample size required to reject the null hypothesis is 256 (128 in each group). With an expected loss of follow-up around 20\%, the sample size was increased to 300 participants in total.

\section{Ethical considerations and adherence to protocol}

This study protocol was approved by the institutional review board (IRB) at King Saud University's College of Medicine, approval number E-17-2607. It is also registered as an International Standard Randomized Control Trial, registration number ISRCTN10857643. All participants will sign a written consent form prior to enrolment in this trial at baseline. For the actual study participants, there will be non-monetary incentives of printed-out visual representations of their progress at follow-up visits.

For fidelity to the protocol, the project manager will oversee the workflow of the intervention, follow-up visits, managerial and data management components, and ensure compliance with the work plan. Furthermore, the IRB at King Saud University's College of Medicine requires 6-monthly study progress reports, projects are monitored by random audits and require the submission of an annual report for protocol approval continuation.

To avoid possible bias, the laboratory staff, biostatistician, health educator, and principal investigator will be blinded to the participant's group allocation until the end of the study. Neither the participants nor the research assistant/project manager responsible for disseminating the text messages will be blinded to the intervention due to its nature.

\section{Discussion}

This trial aims to examine the effect of a TTM based on short messages (text 4 change) towards a lifestyle modification intervention to prevent or delay the onset of T2DM. The intervention will promote a healthy diet and increase in physical activity among subjects with IGT. We are targeting the eligible participants from the HHP study for this trial. The well-trained research team will deliver the intervention according to the TTM stages, to measure behavioral changes in the prediabetic participants. Therefore, the effectiveness of the SMS messages on behavioral change can be tested as proposed and planned.

This trial has been designed to address the scarcity in published studies about the effectiveness of lifestyle modification in preventing the occurrence of diabetes, using SMS as an intervention following the TTM model. Furthermore, the current trial will also provide evidence for progression and risk reduction in this category of participants with IFG or IGT. Applying this new approach to promote the importance of lifestyle modification in prediabetics is expected to delay and/or prevent the development of T2DM in Saudi Arabia. Subsequently, the risk of CV morbidity and mortality will also be reduced. Thus, results from this trial will promote an innovative and high-tech way to decrease the burden of CVD and diabetes management in Saudi Arabia.
Abbreviations

ADA: American Diabetes Association; ATP-III: third adult treatment panel; BMI: Body mass index; C: Contemplation; CDC: Center for Disease Control and Prevention; CV: Cardiovascular; CVD: Cardiovascular diseases;

HbA1c: Glycated hemoglobin; HDL-C: High-density lipoprotein cholesterol; HHP: Heart health promotion study; IFG: Impaired fasting glucose;

IGT: Impaired glucose tolerance; IRB: Institutional review board; LDL-C: Lowdensity lipoprotein cholesterol; MetS: Metabolic syndrome; NCEP: National cholesterol education program; SMS: Short message service; T2DM: Type 2 diabetes mellitus; TTM: Transtheoretical model; US: the United States; WHO: World Health Organization

\section{Acknowledgements}

Special thanks for support by the College of Medicine Research Center, Deanship of Scientific Research, King Saud University Riyadh, Saudi Arabia. We would like to thank Ms. Monica Ronghe (Editing and Proofreading Services, Australia) for editing the final draft of the manuscript.

\section{Authors' contributions}

$\mathrm{AH}$ conceived the study and $L R B$ is the principle investigator. $R Z, M M H, H E$, $A F, T K$, and $Z S$ initiated the study design. $L R B, A H, M M H$ and $R Z$ are grant holders. HE and AF provided statistical expertise in the clinical trial design and AF is conducting the primary statistical analysis. LRB substantively revised and edited the protocol drafts and approved the final version. All authors have read and have approved the final manuscript.

\section{Authors' information}

RZ: Clinical researcher, Cardiac Sciences Department, College of Medicine, King Saud University, Riyadh, Saudi Arabia.

ZS: Associate professor, High Institute of Public Health, Alexandria University, Egypt.

MMH: Lecturer, Community Medicine Unit, Family and Community Medicine Department, College of Medicine, King Saud University, Riyadh, Saudi Arabia. LRB: Assistant professor and clinical epidemiologist, Department of Family and Community Medicine, King Saud University and King Khalid University Hospital, Riyadh, Saudi Arabia.

$\mathrm{AH}$ : Professor, consultant cardiologist and cardiac electrophysiologist, Cardiac Sciences Department, College of Medicine, King Saud University and King Khalid University Hospital, Riyadh, Saudi Arabia.

AF: Associate professor of Public Health, Princess Norah Bint Abdulrahman University, College of Medicine, Riyadh, Saudi Arabia; High Institute of Public Health, Alexandria University, Alexandria, Egypt.

TK: Professor and consultant cardiologist, Cardiac Sciences Department, College of Medicine, King Saud University and King Khalid University Hospital, Riyadh, Saudi Arabia.

HE: Professor, Princess Nourah bint Abdulrahman University, Riyadh, Saudi Arabia; High Institute of Public Health, Alexandria University, Alexandria, Egypt.

\section{Funding}

We have applied for funding, it is pending approval and confirmation. Prospective funders do not have any role or effect on the study design, data collection, analysis, or reporting and results.

\section{Availability of data and materials \\ Not applicable.}

\section{Ethics approval and consent to participate}

This study protocol was approved by the IRB at King Saud University's College of Medicine, approval number E-17-2607. It is also registered as an international standard randomized control trial, registration number ISRCTN10857643; registered on 4th of June 2018. All participants will sign a written consent form prior to enrolment in this trial. 


\section{Consent for publication}

Not applicable.

\section{Competing interests}

The authors declare that they have no competing interests.

\section{Author details}

${ }^{1}$ Cardiac Sciences Department, College of Medicine, King Saud University, Riyadh, Saudi Arabia. ${ }^{2}$ High Institute of Public Health, Alexandria University, Alexandria, Egypt. ${ }^{3}$ Community Medicine Unit, Family and Community Medicine Department, College of Medicine, King Saud University, Riyadh, Saudi Arabia. ${ }^{4}$ Department of Family and Community Medicine, King Saud University and King Khalid University Hospital, Riyadh, Saudi Arabia. ${ }^{5}$ Princess Nourah Bint Abdulrahman University, College of Medicine, Riyadh, Saudi Arabia.

Received: 18 April 2019 Accepted: 8 November 2019

Published online: 21 November 2019

\section{References}

1. Pate RR, Pratt M, Blair SN, Haskell WL, Macera CA, Bouchard C, et al. Physical activity and public health. A recommendation from the Centers for Disease Control and Prevention and the American College of Sports Medicine. JAMA. 1995;273(5):402-7.

2. Mokdad AH, Ford ES, Bowman BA, Dietz WH, Vinicor F, Bales VS, et al. Prevalence of obesity, diabetes, and obesity-related health risk factors, 2001. JAMA. 2003;289(1):76-9.

3. Tirosh A, Shai I, Tekes-Manova D, Israeli E, Pereg D, Shochat T, et al. Normal fasting plasma glucose levels and type 2 diabetes in young men. N Engl J Med. 2005;353(14):1454-62.

4. Meigs JB, Muller DC, Nathan DM, Blake DR, Andres R. The natural history of progression from normal glucose tolerance to type 2 diabetes in the Baltimore longitudinal study of aging. Diabetes. 2003;52(6):1475-84.

5. Dinneen SF, Maldonado D, Leibson CL, Klee GG, Li H, Melton LJ, et al. Effects of changing diagnostic criteria on the risk of developing diabetes. Diabetes Care. 1998;21(9):1408-13.

6. Zhang X, Gregg EW, Williamson DF, Barker LE, Thomas W, Bullard KM, et al. A1c level and future risk of diabetes: a systematic review. Diabetes Care. 2010;33(7):1665-73.

7. Bahijri SM, Jambi HA, Al Raddadi RM, Ferns G, Tuomilehto J. The prevalence of diabetes and Prediabetes in the adult population of Jeddah, Saudi Arabia--A Community-Based Survey. PloS one. 2016;11(4):e0152559.

8. Forouhi NG, Luan J, Hennings S, Wareham NJ. Incidence of type 2 diabetes in England and its association with baseline impaired fasting glucose: the Ely study 1990-2000. Diabet Med. 2007;24(2):200-7.

9. Pan XR, Li GW, Hu YH, Wang JX, Yang WY, An ZX, et al. Effects of diet and exercise in preventing NIDDM in people with impaired glucose tolerance. The Da Qing IGT and diabetes study. Diabetes Care. 1997;20(4):537-44.

10. Ramachandran A, Snehalatha C, Mary S, Mukesh B, Bhaskar AD, Vijay V, et al. The Indian diabetes prevention Programme shows that lifestyle modification and metformin prevent type 2 diabetes in Asian Indian subjects with impaired glucose tolerance (IDPP-1). Diabetologia. 2006;49(2): 289-97.

11. Tuomilehto J, Lindstrom J, Eriksson JG, Valle TT, Hamalainen H, llanneParikka $\mathrm{P}$, et al. Prevention of type 2 diabetes mellitus by changes in lifestyle among subjects with impaired glucose tolerance. N Engl J Med. 2001; 344(18):1343-50.

12. Tudor-Locke C, Bassett DR Jr. How many steps/day are enough? Preliminary pedometer indices for public health. Sports Med (Auckland, NZ). 2004;34(1):1-8.

13. Perry RC, Baron AD. Impaired glucose tolerance. Why is it not a disease? Diabetes Care. 1999;22(6):883-5.

14. Tominaga M, Eguchi H, Manaka H, Igarashi K, Kato T, Sekikawa A. Impaired glucose tolerance is a risk factor for cardiovascular disease, but not impaired fasting glucose, The Funagata Diabetes Study. Diabetes Care. 1999;22(6): 920-4.

15. Koskinen P, Manttari M, Manninen V, Huttunen JK, Heinonen OP, Frick MH. Coronary heart disease incidence in NIDDM patients in the Helsinki heart study. Diabetes Care. 1992;15(7):820-5.

16. WHO-Global-Report-DM. Global report on diabetes.http://www.who.int/ diabetes/global-report/en/.Date of access Jan 2018.
17. Badran M, Laher I. Type II diabetes mellitus in Arabic-speaking countries. Int J Endocrinol. 2012;2012:902873

18. Mokdad AH, Tuffaha M, Hanlon M, El Bcheraoui C, Daoud F, Al Saeedi M, et al. Cost of diabetes in the Kingdom of Saudi Arabia, 2014. J Diabetes Metab. 2015;6:8.

19. Al-Rubeaan K, Al-Manaa HA, Khoja TA, Ahmad NA, Al-Sharqawi AH, Siddiqui $K$, et al. Epidemiology of abnormal glucose metabolism in a country facing its epidemic: SAUDI-DM study. J Diabetes. 2015;7(5):622-32.

20. Alzeidan R, Rabiee F, Mandil A, Hersi A, Fayed A. Non-communicable disease risk factors among employees and their families of a Saudi University: An epidemiological study. PLoS One. 2016;11(11):e0165036.

21. Haller D, Sanci L, Sawyer S, Coffey C, Patton G. R U OK 2 TXT 4 RESEARCH?--feasibility of text message communication in primary care research. Aust Fam Physician. 2006;35(3):175-6.

22. Fjeldsoe BS, Marshall AL, Miller YD. Behavior change interventions delivered by mobile telephone short-message service. Am J Prev Med. 2009;36(2): 165-73.

23. Puri-Mirza A. Number of smartphone users in Saudi Arabia from 2017 to 2023. https://www.statista.com/statistics/494616/smartphone-users-in-saudiarabia/. date of access sep 2019.

24. Prochaska JO, DiClemente CC. Stages and processes of self-change of smoking: toward an integrative model of change. J Consult Clin Psychol. 1983;51(3):390-5.

25. Bobrow K, Farmer AJ, Springer D, Shanyinde M, Yu LM, Brennan T, et al. Mobile phone text messages to support treatment adherence in adults with high blood pressure (SMS-text adherence support [StAR]): a single-blind, Randomized Trial. Circulation. 2016;133(6):592-600.

26. Farmer AJ, Prevost AT, Hardeman W, Craven A, Sutton S, Griffin SJ, et al. Protocol for SAMS (support and advice for medication study): a randomised controlled trial of an intervention to support patients with type 2 diabetes with adherence to medication. BMC Fam Pract. 2008;9(1):20.

27. Wong CK, Jiao FF, Siu SC, Fung CS, Fong DY, Wong KW, et al. Costeffectiveness of a short message service intervention to prevent type 2 diabetes from impaired glucose tolerance. J Diabetes Res. 2016;2016: 1219581.

28. Gokbayrak NS, Paiva AL, Blissmer BJ, Prochaska JO. Predictors of relapse among smokers: transtheoretical effort variables, demographics, and smoking severity. Addict Behav. 2015;42:176-9.

29. Menezes MC, Mingoti SA, Cardoso CS, de Deus MR, Lopes AC. Intervention based on Transtheoretical model promotes anthropometric and nutritional improvements - a randomized controlled trial. Eat Behav. 2015;17:37-44.

30. Zhu LX, Ho SC, Sit JW, He HG. The effects of a transtheoretical model-based exercise stage-matched intervention on exercise behavior in patients with coronary heart disease: a randomized controlled trial. Patient Educ Couns. 2014;95(3):384-92.

31. Lee JE, Lee DE, Kim K, Shim JE, Sung E, Kang J-H, et al. Development of tailored nutrition information messages based on the transtheoretical model for smartphone application of an obesity prevention and management program for elementary-school students. Nutr Res Pract. 2017; 11(3):247-56.

32. Marcus BH, Bock BC, Pinto BM, Forsyth LH, Roberts MB, Traficante RM. Efficacy of an individualized, motivationally-tailored physical activity intervention. Ann Behav Med. 1998;20(3):174-80.

33. Alberti KG, Zimmet PZ. Definition, diagnosis and classification of diabetes mellitus and its complications. Part 1: diagnosis and classification of diabetes mellitus. Provisional report of a WHO consultation. Diabet Med. 1998;15(7):539-53.

34. Spencer L, Adams TB, Malone S, Roy L, Yost E. Applying the transtheoretical model to exercise: a systematic and comprehensive review of the literature. Health Promot Pract. 2006;7(4):428-43.

35. WHO-HBA1C. Use of Glycated Haemoglobin ( $\mathrm{HbA1c}$ ) in the Diagnosis of Diabetes Mellitus:abbreviated report of a WHO consultation .WHO: Geneva 2011 http://www.who.int/diabetes/publications/report-hba1c_2011.pdf. Date of access May 2014.

36. Gordon DJ, Probstfield JL, Garrison RJ, Neaton JD, Castelli WP, Knoke JD, et al. High-density lipoprotein cholesterol and cardiovascular disease. Four prospective American studies. Circulation. 1989;79(1):8-15.

37. US NCEP A, III. Third report of the National Cholesterol Education Program (NCEP) expert panel on detection, evaluation, and treatment of high blood cholesterol in adults (adult treatment panel III) final report. Circulation. 2002; 106(25):3143-421. 
38. Grundy SM, Cleeman Jl, Merz CN, Brewer HB Jr, Clark LT, Hunninghake DB, et al. Implications of recent clinical trials for the National Cholesterol Education Program Adult Treatment Panel III guidelines. Circulation. 2004; 110(2):227-39.

39. activity WH-p. Physical activity fact sheet N 385 unpdated Feb 2018 https:// www.who.int/news-room/fact-sheets/detail/physical-activity . Date of access Sept. 2019.

40. diet wh. healthy diet http://www.who.int/mediacentre/factsheets/fs394/en/ updated October 2018. Date of access Sept 2019.

41. WHO-BMI. Global Database on Body Mass Index http://apps.who.int/bmi/ index.jsp?introPage=intro_3.html. 2004. Date of access April 2014.

42. Grundy SM, Cleeman JI, Daniels SR, Donato KA, Eckel RH, Franklin BA, et al. Diagnosis and management of the metabolic syndrome: an American Heart Association/National Heart, Lung, and Blood Institute scientific statement. Circulation. 2005:112(17):2735-52.

43. de VriesRAJ TKP, Kwint S, Drossaert CHC, Crowd-Designed EV. Motivation: motivational messages for exercise adherence based on behavior change theory. San Jose: University of Twente; 2016. p. 297-308. https://dl.acm.org/ citation. ffm?doid=2858036.2858229. Accessed May 2017.

44. de Vries RAJ, Truong KP, Zaga C, Li J, Evers V. A word of advice: how to tailor motivational text messages based on behavior change theory to personality and gender. Pers Ubiquit Comput. 2017;21(4):675-87.

\section{Publisher's Note}

Springer Nature remains neutral with regard to jurisdictional claims in published maps and institutional affiliations.

Ready to submit your research? Choose BMC and benefit from:

- fast, convenient online submission

- thorough peer review by experienced researchers in your field

- rapid publication on acceptance

- support for research data, including large and complex data types

- gold Open Access which fosters wider collaboration and increased citations

- maximum visibility for your research: over $100 \mathrm{M}$ website views per year

At $\mathrm{BMC}$, research is always in progress.

Learn more biomedcentral.com/submissions 